\title{
Konsum von Kokain vor, in und nach der heroingestützten Behandlung in der Schweiz
}

\author{
Patrick Gschwend ${ }^{1}$ \\ Susanne Eschmann ${ }^{1}$ \\ Franziska Güttinger ${ }^{1}$ \\ Simone Rey-Riek ${ }^{1}$ \\ Jürgen Rehm ${ }^{1,2,3}$ \\ Ambros Uchtenhagen ${ }^{1}$
}

\author{
Cocaine Consumption Before, In Course of, \\ and After Heroinassisted Treatment in Switzerland
}

\section{Zusammenfassung}

Anliegen: Der vorliegende Artikel gibt einen Überblick über das Ausmaß des Kokainkonsums von 1994 bis 2001 von Patienten der heroingestützten Behandlung in der Schweiz vor ihrem Eintritt, im Behandlungsverlauf und nach deren Austritt.

Methode: Zum Kokainkonsum der Patienten liegen einerseits objektive Daten von Urinproben, andererseits Selbstangaben der Patienten zu ihrem Substanzkonsum zu verschiedenen Zeitpunkten (bis 6 Jahre nach Behandlungsbeginn) vor.

Ergebnisse: Der Konsum von Kokain nimmt in der heroingestützten Behandlung bereits innerhalb von 6 Monaten ab und liegt auch nach Austritt auf tieferem Niveau als bei Eintritt. Die Prävalenz des Kokainkonsums bei Behandlungsbeginn hat von 1994 bis 2001 abgenommen und ist bei den Patienten in der Stadt Zürich höher als bei Patienten der restlichen Behandlungsstellen. Die letzte Stichtagserhebung an einer kleinen Stichprobe zeigt einen Anstieg des Kokainkonsums von in Behandlung stehenden Personen.

Schlussfolgerungen: Kokainkonsum bleibt in der heroingestützten Behandlung weiterhin ein Problem. Maßnahmen gegen den Neben- und namentlich den Kokainkonsum haben deshalb weiterhin hohe Priorität.

\section{Schliuisselwörter}

Opiatabhängigkeit · Heroinverschreibung · Kokain · Schweiz

\section{Abstract}

Objective: This paper gives an overview of cocaine consumption among patients in heroin-assisted treatment in Switzerland at treatment entry, during treatment and at discharge. It includes patients entering this form of treatment from 1994 to 2001.

Methods: At entry, urinalysis and self-report for the patients; at different follow-up times (up to 6 years), self-reports, in part corroborated by urinalysis; additionally random urinalysis testing on one set day.

Results: Cocaine consumption decreased within the first 6 months in heroin-assisted treatment and stays at a lower level even after treatment discharge. Prevalence of cocaine consumption at entry has been decreasing from 1994 to 2001, and has been higher in town of Zurich. The last small study showed increases in prevalence among persons in treatment.

Conclusions: Cocaine consumption remains a problem in heroin-assisted treatment. Thus, measures to cope with this problem continue to have high priority.

Key Words

Opiate Dependency $\cdot$ Heroin Prescription $\cdot$ Cocaine $\cdot$ Switzerland

${ }^{1}$ Institut für Suchtforschung, Zürich, Schweiz

${ }^{2}$ Centre for Addiction and Mental Health, Toronto, Canada

${ }^{3}$ Public Health Sciences, University of Toronto, Canada 
Die heroingestützte Behandlung in der Schweiz war von 1994 bis 1996 als breites wissenschaftliches Forschungsprojekt konzipiert worden [1,2]. Heute ist sie eine gut dokumentierte Routinebehandlung [3], die häufig mit zusätzlichen spezifischen Forschungsprojekten verbunden wird. Untersuchungen zum Substanzmittelkonsum in der heroingestützten Behandlung in der Schweiz zeigen, dass die Patienten bei Eintritt häufig auch andere psychoaktive Substanzen und im besonderen Kokain konsumierten oder von ihnen abhängig waren [1].

Opioidabhängige benutzen Kokain aus verschiedenen Gründen: um den sedativen Effekt von Opiaten zu antagonisieren, eine Euphorie zu erzeugen, die von Opiaten unabhängig ist, und Opioidentzugserscheinungen zu unterdrücken [4]. Der Nebenkonsum von illegalen Substanzen und damit auch der Kokainkonsum stehen aber den Zielen der heroingestützten Behandlung - wie bei anderen Behandlungsangeboten für Drogenabhängige - entgegen: Er beeinträchtigt die Verbesserung des physischen und psychischen Gesundheitszustands und erschwert die Distanzierung vom Drogenmilieu und dadurch die soziale Reintegration [5].

Die Behandlung der Kokainabhängigkeit insbesondere bei Opioidabhängigen ist sehr schwierig [6,7]. Immerhin werden heute beispielsweise kognitiv-verhaltenstherapeutische Methoden mit bedingtem Erfolg eingesetzt [8].

Die Wirksamkeit der Methadonbehandlung auf die Reduktion des Kokainnebenkonsums von Opioidabhängigen ist fragwürdig und wird unterschiedlich beurteilt. Einige Autoren kommen zum Schluss, dass Methadon unter Umständen vor Kokainkonsum schützt $[9,10]$. Dagegen bezeichnen andere Methadon als unwirksam, was den Kokainkonsum anbelangt [11 - 13] (für einen Überblick siehe [14]).

Die vorliegende Arbeit gibt Auskunft über das Ausmaß des Kokainkonsums von Patienten bei Eintritt in die heroingestützte Behandlung in der Zeit von 1994 bis 2001 sowie über die Prävalenz des Kokainkonsums in und den Verlauf während und nach dieser Behandlung. Gleichzeitig wird untersucht, ob Kokainkonsum bei Patienten in der Stadt Zürich häufiger ist, ob sich die Rate des Kokainkonsums bei Eintritt seit 1994 verändert hat, ob sie während der heroingestützten Behandlung zurückgeht und auch nach Austritt geringer ist als bei Behandlungsbeginn. Weiter wird die Annahme geprüft, dass der Beikonsum nach dem Wochenende oder nach Auszahlung von Geldern (z.B. Löhne, Renten oder Fürsorgegelder) erhöht ist.

\section{Material und Methoden}

Seit Einführung der heroingestützten Behandlung in der Schweiz 1994 wurde bei den Patienten eine Reihe von Untersuchungen zum Beikonsum durchgeführt. Diese Arbeit stützt sich auf Daten der Eintritts- und Verlaufsbefragungen nach 6, 12 und 18 Monaten [1,2], einer Nachbefragung nach 6 Jahren [15] sowie einer im 2001 durchgeführten Pilotstudie zur Erfassung des Nebenkonsums.

\section{Eintrittsbefragungen}

Bei Eintritt wurden alle 2051 Patientinnen und Patienten (Stand 30.11.2001) ausführlich zu ihrer sozialen und gesundheitlichen Situation befragt. Bezüglich Kokainkonsums wurden die Eintretenden von 1994 bis 1996 und ab November 2000 nach dem Konsum in den letzten 30 Tagen gefragt. Von 1998 bis Oktober 2000 bezog sich der Zeitraum bei dieser Frage auf die letzten 6 Monate. 1997 waren aus gesetzlichen Gründen keine Neuaufnahmen möglich [16].

In dieser Untersuchung wurden wegen der Vergleichbarkeit der Häufigkeit von Kokainkonsum in den einzelnen Jahren nur die Daten von denjenigen 1417 Personen berücksichtigt, die zum Kokainkonsum in den letzten 30 Tagen befragt wurden.

\section{Verlaufsbefragungen}

Bei denjenigen 366 Personen, die zwischen 1994 und 1996 in die heroingestützte Behandlung eingetreten waren, fand nach 6, 12 und 18 Monaten eine Nachbefragung statt, sofern sie sich zum Befragungszeitpunkt noch in Behandlung befanden [1,2]. Die Selbstangaben zum Kokainkonsum wurden teilweise mit Urinproben überprüft und zeigten eine relativ hohe Übereinstimmung mit den Resultaten der Laboruntersuchungen [17].

Im Zeitraum von November 2000 bis März 2001 fand außerdem eine Nachbefragung bei den ersten 366 Patientinnen und Patienten der heroingestützten Behandlung in der Schweiz rund 6 Jahre nach Behandlungsbeginn statt. Davon waren zum Befragungszeitpunkt 43 Personen verstorben. Die lebenden Patienten wurden erneut ausführlich befragt, unabhängig davon, ob sie sich noch in Behandlung befanden oder bereits ausgetreten waren [15]. Bei dieser Untersuchung nahmen 112 der 175 ausgetretenen (64,0\%) und 132 der 148 in Behandlung stehenden Patienten $(89,2 \%)$ teil. Es wurden keine Urinuntersuchungen durchgeführt.

\section{Beikonsumerhebung}

Ende September 2001 fand außerdem eine Pilotstudie zur Erfassung des Beikonsums in der heroingestützten Behandlung statt. Im Rahmen dieser Untersuchung wurde von einer Stichprobe von 42 in Behandlung stehenden Patienten der Substanzenkonsum mit Urinproben überprüft. Die untersuchte Stichprobe wurde aus je 2 zufällig ausgewählten Patienten der 21 Behandlungsstellen gebildet, die bereit waren, an der Untersuchung mitzumachen. 10 Personen, die angefragt wurden, verweigerten ihre Teilnahme.

Bei der schließlich rekrutierten Stichprobe handelt es sich um 33 Männer (78,6\%) und 9 Frauen (21,4\%) mit einem Durchschnittsalter von 37,1 Jahren (SD: 5,8). Bis zur Untersuchung betrug der letzte Aufenthalt in der heroingestützten Behandlung im Durchschnitt 3,7 Jahre (SD: 2,5). Die Patienten waren vor dieser Erhebung im Durchschnitt also schon relativ lange Zeit in Behandlung.

\section{Statistik}

Die Analyse der genannten Daten erfolgte am Institut für Suchtforschung mit dem Statistikpaket SPSS 10.0 für Macintosh. Die Vergleiche erfolgten je nach Skalenniveau und Zellbesetzung mittels Cochran's Q-, $\chi^{2}$ - und Fisher's-Exact-Test. Als Signifikanzniveau wurde $\mathrm{p}<0,05$ gewählt. 


\section{Verlauf des Kokainkonsums während und nach der heroingestuitzten Behandlung}

Prävalenz des Kokainkonsums bei den Eintritten 1994 - 2001 Die Mehrzahl der eintretenden Personen konsumierte im Monat vor Beginn der heroingestützten Behandlung (selten, gelegentlich oder täglich) Kokain. Der Anteil der Personen, die keinen Konsum berichteten, stieg zwischen 1994 bis 2001 von 19,8\% auf $33,7 \%$.

Von 1994 bis 1996 waren in Stadtzürcher Behandlungsstellen Eintretende vor ihrem Behandlungsbeginn deutlich weniger abstinent von Kokain als die Eintritte der restlichen Zentren für heroingestützte Behandlung in der Schweiz. Danach gleichen sich die Abstinenzraten an (Tab.1).

\section{Prävalenz des Kokainkonsums in der heroingestützten Behandlung (Pilotstudie zur Erhebung des Beikonsums)}

Bei der Beikonsumerhebung im Jahr 2001 wurden 31,7\% (13 von 41) Urinproben positiv auf Kokain getestet. Unter der Annahme (worst-case), dass alle 10 Verweigerer auch Kokain konsumierten, beträgt der Anteil 45,1\% (23 von 51).

Wochentag und Erhalt von Geld zeigen in dieser Stichprobe keinen Einfluss auf das Beikonsumverhalten. Ferner zeigte sich, dass das Personal der Behandlungsstellen Abstinenz der Patienten von Kokain besser einschätzen kann als Konsum. Von 26 Personen mit einer negativen Urinprobe wurden 24 (92,3\%) vorausgesagt (Tab. 2). Dagegen wurde der Kokainkonsum bei Patienten mit positiver Urinprobe in rund zwei Dritteln der Fälle im Voraus richtig eingeschätzt.
Tab. 3 gibt den Verlauf des (fast) täglichen Kokaingebrauchs der ersten Patienten der heroingestützten Behandlung in der Schweiz wieder. Die Einteilung in „Patientinnen“ und „Austritte“ bezieht sich dabei auf den Behandlungsstatus rund 6 Jahre nach Eintritt in die Therapie. Zum Befragungszeitpunkt waren 148 der 323 lebenden Personen (45,8\%) weiterhin oder wieder in Behandlung [15].

Sowohl bei den Patienten als auch bei den Ausgetretenen ist der Konsum von Kokain 6 Jahre nach Beginn der heroingestützten Behandlung hochsignifikant geringer als zur Zeit des Eintritts.

In der Gruppe der „Austritte“ sind diejenigen 18 Personen enthalten, die bei der 6-Jahres-Verlaufsbefragung angaben, in den letzten 6 Monaten weder Opioide noch Kokain konsumiert zu haben und sich auch nicht in einer Substitutionsbehandlung mit z.B. Methadon zu befinden [15]. Der Gebrauch von Kokain ist bei den ausgetretenen Patienten dennoch während des gesamten Behandlungsverlaufs weiter verbreitet als in der Gruppe der in Behandlung stehenden Personen.

\section{Diskussion}

\section{Prävalenz des Kokainkonsums bei Eintretenden in der Zeit} von 1994 bis 2001

Von 1994 an hat bei den Eintretenden in die heroingestützte Behandlung der tägliche Kokainkonsum vor Behandlungsbeginn abgenommen. Der höhere Anteil zu Beginn lässt sich mindestens

Tab. 1 Häufigkeit des Kokainkonsums von Eintretenden in die heroingestützte Behandlung von 1994 bis 2001 in Zürich und der restlichen Schweiz ( $n=1417$ : 354 Patienten von Stadtzürcher und 1063 von anderen Behandlungsstellen) ${ }^{1}$

\begin{tabular}{|c|c|c|c|c|c|}
\hline & $\begin{array}{l}\text { untersuchte Eintritte } \\
1994 \\
(n=389)\end{array}$ & $\begin{array}{l}\text { untersuchte Eintritte } \\
1995 \\
(n=545)\end{array}$ & $\begin{array}{l}\text { untersuchte Eintritte } \\
1996 \\
(n=248)\end{array}$ & $\begin{array}{l}\text { untersuchte Eintritte } \\
2000 \\
(n=30)^{1}\end{array}$ & $\begin{array}{l}\text { untersuchte Eintritte } \\
2001 \\
(n=205)\end{array}$ \\
\hline kein Konsum & $19,8 \%$ a & $26,3 \% \mathrm{~b}$ & $30,0 \% c$ & $25,0 \% d$ & $33,7 \%$ e \\
\hline $\begin{array}{l}\text { Anzahl Patienten } \\
\text { ZH Zentren }\end{array}$ & 150 & 108 & 50 & 7 & 39 \\
\hline $\begin{array}{l}\text { kein Konsum } \\
\text { ZH Patienten }\end{array}$ & $11,6 \%^{f}$ & $13,3 \% \mathrm{~g}$ & $11,1 \% \mathrm{~h}$ & $33,3 \% i$ & $29,7 \%$ j \\
\hline
\end{tabular}

Im Jahr 1997 waren keine Neuaufnahmen in die heroingestützte Behandlung möglich.

1 Von 1998 bis Oktober 2000 bezog sich der Befragungszeitraum auf die letzten 6 Monate. Wegen der Vergleichbarkeit wurden diese Daten für diese Auswertung nicht berücksichtigt.

Fehlende Werte: a5, b21, c11, d9, e18, f3, g10, h5, i1, j2.

Tab. 2 Vergleich der Laborbefunde und der Einschätzung der Therapeuten bez. Kokainkonsum der betreffenden Patienten in den letzten 2 Tagen $\left(\mathrm{n}=37, \mathrm{mv}=5^{1}\right)$

\begin{tabular}{lcc}
\hline & Patienten mit positiver UP (n= 11) & Patienten mit negativer UP (n= 26) \\
\hline & $7(63,6 \%)$ & $2(7,7 \%)$ \\
\hline richtige Einschätzung & $4(36,4 \%)$ & $24(92,3 \%)$ \\
\hline falsche Einschätzung & Fisher's Exact Test: $\mathrm{P}=0,001$ \\
\hline $\begin{array}{l}\text { Bei einer Person war die Urinprobe nicht richtig beschriftet und wurde deshalb vom Labor nicht getestet. Von } 4 \text { anderen Personen lagen keine Einschätzungen zum } \\
\text { Kokainkonsum vor. }\end{array}$
\end{tabular}

\footnotetext{
Kokainkonsum vor.
} 
teilweise damit erklären, dass sich in der ersten Versuchsphase viele meldeten, die auf eine Kokainverschreibung hofften, da diese grundsätzlich Teil des Versuches sein durfte. Ob sich im Verlauf der Zeit auch die Charakteristik der Eintretenden verändert hat und heute weniger belastete Personen in die heroingestützte Behandlung eintreten, kann mit den vorliegenden Daten nicht beurteilt werden.

In den 90er Jahren war Kokain in Zürich gut verfügbar $[18,19]$. Dies war wahrscheinlich mit ein Grund für die von 1994 bis 1996 höhere Prävalenz von (täglichem) Kokainkonsum bei in Stadtzürcher Behandlungsstellen Eintretenden. Der Rückgang des selbst berichteten Konsums im Jahr 2000 und 2001 ist (vermutlich) nicht auf eine Verknappung dieser Droge zurückzuführen. Eine Erklärung dürfte wiederum die von vielen Eintritten anfänglich erhoffte Kokainverschreibung sein (siehe oben). Die weiteren Ursachen wären noch zu untersuchen.

\section{Verlauf des Kokainkonsums während und nach der heroingestützten Behandlung}

Bei den Patientinnen und Patienten der heroingestützten Behandlung, die mindestens 18 Monate in Therapie waren, findet sich entsprechend früheren Befunden $[1,2]$ bereits nach 6 Monaten ein deutlicher Rückgang des Kokaingebrauchs. Der Verlauf des Kokainkonsums in der Gesamtstichprobe wurde zudem mit einer strengeren Methode geschätzt (siehe Tab. 3, Spalte „Schätzung Kokainkonsum plausible Annahmen“). Diese Analyse basiert auf den Hypothesen, dass 10\% der Selbstangaben falsch negativ sind, dass die Häufigkeit des täglichen Kokainkonsums vor Behandlungsbeginn bei Patienten, für die bei Eintritt keine Daten vorhanden waren, dem der Antwortenden entspricht und dass Personen mit fehlenden Werten ab dem sechsten Behandlungsmonat (trotz Last-Observation-Carried-Forward-Technik) täglich Kokain konsumieren. Unter diesen Annahmen zeigt sich ein knapp nicht signifikanter Rückgang des täglichen Kokainkonsums bei der Gesamtheit der Eintretenden über die gesamte Beobachtungsperiode (Tab. 3).
Da Kokain bis auf eine kurze Versuchsphase (kokainhaltige Zigaretten) in der heroingestützten Behandlung nie substituiert wurde, kommt der Rückgang aufgrund anderer Faktoren zustande [20]. Eine bedeutende Ursache dürfte der große Aufwand sein, den die Behandlungsstellen unter anderem für die Reduktion des Nebenkonsums ihrer Patienten betreiben.

Der in der Beikonsumerhebung von 2001 identifizierte Anteil von 31,7\% Kokainkonsumierenden unter den Patienten steht den tieferen Werten, die in den früheren Verlaufsuntersuchungen gefunden wurden, entgegen. Möglicherweise hat der Kokainkonsum in den letzten Jahren vor dem Hintergrund tiefer Schwarzmarktpreise [21] und allgemein steigender Beliebtheit zugenommen. Bei der Bewertung dieser Angaben muss auch bedacht werden, dass die Stichprobe relativ klein war und die entsprechenden Konfidenzintervalle noch relativ weit sind und so Schwankungen zulassen.

\section{Schlussfolgerungen}

In der heroingestützten Behandlung zeigt sich bei den in Behandlung stehenden Personen bereits innerhalb von 6 Monaten ein Rückgang des Kokaingebrauchs. Trotz des beobachteten Rückgangs besteht selbst bei Patienten, die länger in Behandlung stehen, nach wie vor unerwünschter Beikonsum von Kokain. Einer der Schwerpunkte der Behandlung muss deshalb weiterhin das Ergreifen von Maßnahmen zur Reduktion dieses Beikonsums sein.

\section{Danksagung}

Diese Arbeit entstand im Auftrag des Schweizer Bundesamtes für Gesundheit BAG (Vertrag Nr. 00.001494).

Für die Unterstützung dieser Studie möchten wir uns bei den Behandlungsstellen und dem Bundesamt für Gesundheit ganz herzlich bedanken.

Tab. 3 Täglicher oder fast täglicher Kokainkonsum von Patienten, die nach 6 Jahren noch in der heroingestützten Behandlung oder ausgetreten waren $(n=323)$

\begin{tabular}{|c|c|c|c|c|}
\hline & $\begin{array}{l}\text { Patienten } \\
(n=148)\end{array}$ & $\begin{array}{l}\text { Austritte } \\
(\mathrm{n}=175)\end{array}$ & $\begin{array}{l}\text { gesamte Kohorte } A \\
(n=323)\end{array}$ & $\begin{array}{l}\text { Schätzung Kokainkonsum } \\
\text { plausible Annahmen }(n=323)\end{array}$ \\
\hline T0: vor Eintritt & $38(27,1 \%)^{\mathrm{a}}$ & $57(34,5 \%)^{b}$ & $95(31,1 \%)^{c}$ & $100(31,0 \%)$ \\
\hline T1: nach 6 Monaten & $16(10,9 \%)^{d}$ & $39(23,2 \%)^{e}$ & $55(17,5 \%)^{f}$ & $88(27,2 \%)$ \\
\hline T2: nach 12 Monaten & $12(8,1 \%)^{g}$ & $36(21,4 \%)^{\mathrm{h}}$ & $48(15,2 \%)^{i}$ & $81(25,1 \%)$ \\
\hline T3: nach 18 Monaten & $14(9,5 \%)^{j}$ & $37(22,0 \%)^{k}$ & $51(16,1 \%)^{\prime}$ & $84(26,0 \%)$ \\
\hline T4: nach 6 Jahren & $9(6,1 \%)^{m}$ & $26(15,2 \%)^{n}$ & $35(11,0 \%)^{\circ}$ & $67(20,7 \%)$ \\
\hline $\begin{array}{l}\text { Veränderung von T0 zu T4: } \\
\text { statistisches Verfahren }\end{array}$ & $\begin{array}{l}\text { Cochran's } Q=26,0 \\
d f=1, p<0,001\end{array}$ & $\begin{array}{l}\text { Cochran's } Q=23,4, \\
d f=1, p<0,001\end{array}$ & $\begin{array}{l}\text { Cochran's } Q=49,3 \\
d f=1, p<0,001\end{array}$ & $\begin{array}{l}C h i^{2}=9,2, d f=4 \\
p=0,057\end{array}$ \\
\hline
\end{tabular}

Die Zahlen entsprechen Personen (\% ist der Anteil Personen, die einen gültigen Wert haben). Basierend auf der „last observation carried forward“-Technik. Fehlende Werte: a8, b10, c18, d23, e103, f126, g23, h105, i128, j24, k102, I126, m22, n63, o85. 


\section{Literatur}

${ }^{1}$ Betäubungsmittelverschreibung an Heroinabhängige - Wichtigste Resultate der Schweizerischen Kohortenstudie In: Uchtenhagen A, Dobler-Mikola A, Steffen T, Gutzwiller F, Blättler R, Pfeifer S (Hrsg).. Basel: Karger 2000

${ }^{2}$ Rehm J, Gschwend P, Steffen T, Gutzwiller F, Dobler-Mikola A, Uchtenhagen A. Feasibility, safety, and efficacy of injectable heroin prescription for refractory opioid addicts: a follow-up study. Lancet 2001; 385: $1417-1420$

${ }^{3}$ Gschwend P, Rehm J, Lezzi S, Gutzwiller F, Uchtenhagen A. Development of a monitoring system for heroin assisted treatment in Switzerland. Soz Präventivmed; in press

${ }^{4}$ Sourgens H. „Drug interactions“: Gefährdung der Substitution In: Jellinek C, Westermann B, Bellmann GU (Hrsg). Beigebrauch - Offene Grenzen der Substitution. Weinheim: Deutscher Studien Verlag 2000; $57-63$

${ }^{5}$ Gölz J. Nebenkonsum und Beendigung der Substitution, Behandlungsabbruch und Konsequenzen In: Jellinek C, Westermann B, Bellmann GU (Hrsg). Beigebrauch - Offene Grenzen der Substitution. Weinheim: Deutscher Studien Verlag 2000; 43-49

${ }^{6}$ Kolar AF, Brown BS, Weddington WW, Ball JC. A treatment crisis: Cocaine use by clients in methadone maintenance programs. J Subst Abuse Treat 1992; 7: $101-107$

${ }^{7}$ Seidenberg A, Honegger U. Methadon, Heroin und andere Opioide: Medizinisches Manual für die ambulante opioidgestützte Behandlung Bern: Hans Huber 1998

${ }^{8}$ Rosenblum A, Magura S, Foote J, Palij M, Handelsman L, Lovejoy M, Stimmel B. Treatment intensity and reduction in drug use for cocainedependent methadone patients: a dose-response relationship. J Psychoactive Drugs 1995; 27: 151-159(2)

${ }^{9}$ Des Jarlais DC, Wenston J, Friedman SR, Sotheran JL, Maslansky R, Marmor M. Crack cocaine use in a cohort of methadone maintenance patients. J Subst Abuse Treat 1992; 9: 319-325

${ }^{10}$ Verthein U, Degkwitz P, Haasen C, Raschke P, Krausz M. Die Substitutionsbehandlung Opiatabhängiger mit Codein/Dihydrocodein und Methadon - ein Kontrollgruppenvergleich. Sucht 1996; 42: 108-117 (2)
${ }^{11}$ Condelli WS, Fairbank JA, Dennis ML, Rachal JV. Cocaine use by clients in methadone programs: significance, scope, and behavioral interventions. J Subst Abuse Treat 1991; 8: 203-212

${ }^{12}$ Avants SK, Margolin A, Kosten TR. Cocaine abuse in methadone maintenance programs: integrating pharmacotherapy with psychosocial interventions. J Psychoactive Drugs 1994; 28: 137-146 (2)

${ }^{13}$ Kosten TR, Rounsaville BJ, Kleber HD. A 2.5-year follow-up of cocaine use among treated opioid addicts. Have our treatments helped? Arch Gen Psychiatry 1987; 44 (3): $281-284$

${ }^{14}$ Fischer B, Kirst M, Rehm J, Marsh D, Bondy S, Tyndall M. The phenomenon of so-called „other drug use“ among opiate addicts in the North American context: evidence, consequences, questions In: Jellinek C, Westermann B, Bellmann GU (Hrsg). Beigebrauch - Offene Grenzen der Substitution. Weinheim: Deutscher Studien Verlag 2000; 95- 117

${ }^{15}$ Gschwend P, Güttinger F, Schulte B, Steffen T, Rehm J, Uchtenhagen A. Die Wirksamkeit der Heroin gestützten Behandlung auf den Konsum illegaler Substanzen: Eine 6-Jahres Katamnese. Soz Präventivmed; submitted

${ }^{16}$ Steffen T, Kaufmann B, Blättler R, Dobler-Mikola A, Gutzwiller F, Uchtenhagen A. Die heroingestützte Behandlung von Opiatabhängigen - bisherige und aktuelle Forschungsschwerpunkte. Gesundheitswesen 1999; 61: 407-412

17 Blättler R, Dobler-Mikola A, Steffen T, Uchtenhagen A. Decreasing intravenous cocaine use in opiate users treated with prescribed heroin. Soz Präventivmed; in press

${ }^{18}$ Meier Kressig M, Nydegger Lory B, Schumacher C. Nutzen niedrigschwelliger Drogenarbeit am Beispiel der Stadt Zürich. Schlussbericht der Gesamtevaluation der niedrigschwelligen Drogenhilfe in der Stadt Zürich. Zürich: Institut für Suchtforschung 1996

${ }^{19}$ Nydegger Lory B. Transaktionen und Strukturen des Kleinhandels von Heroin und Kokain in Zürich. Zürich: Institut für Suchtforschung 1999

${ }^{20}$ Moldovanyi A. Heroingestützte Behandlung und Beikonsum In: Jellinek C, Westermann B, Bellmann GU (Hrsg). Beigebrauch - Offene Grenzen der Substitution. Weinheim: Deutscher Studien Verlag 2000; $133-140$

${ }^{21}$ Ammann D, Marti M. Kokain - Drogen - Voll auf einer Linie. FactsMagazin 2000; 45: $106 \mathrm{ff}$ 